\section{Monitoring of clonal evolution of acute myeloid leukemia identifies the leukemia subtype, clinical outcome and potential new drug targets for post-remission strategies or relapse}

\author{
Esther Onecha, ${ }^{1,2,3}$ Inmaculada Rapado,, 1,2,3,4 María Luz Morales,, 1,2,3 \\ Gonzalo Carreño-Tarragona, ${ }^{1,2}$ Pilar Martinez-Sanchez, ${ }^{1,2,3}$ Xabier Gutierrez, ${ }^{1}$ \\ José María Sánchez Pina, ${ }^{1}$ María Linares, ${ }^{1,2,3}$ Miguel Gallardo, ${ }^{1,2,3}$ \\ Joaquín Martinez-López, ${ }^{1,2,3,4,5}$ and Rosa Ayala ${ }^{1,2,3,4,5}$ \\ ${ }^{1}$ Hematology Department, Hospital Universitario 12 de Octubre; ${ }^{2}$ Instituto de \\ Investigacion Hospital 12 de Octubre, Imas $12 ;{ }^{3} \mathrm{Hematological} \mathrm{Malignancies} \mathrm{Clinical}$ \\ Research Unit, CNIO; ${ }^{4} \mathrm{CIBERONC}$, Instituto Carlos III and ${ }^{5}$ Complutense University of \\ Madrid, Madrid, Spain
}

\section{ABSTRACT}

حestar n cases of treatment failure in acute myeloid leukemia (AML), the utility of mutational profiling in primary refractoriness and relapse is not Lestablished. We undertook a perspective study using next-generation sequencing (NGS) of clinical follow-up samples $(\mathrm{n}=91)$ from 23 patients with AML with therapeutic failure to cytarabine plus idarubicin or fludarabine. Cases of primary refractoriness to treatment were associated with a lower number of DNA variants at diagnosis than cases of relapse (median 1.67 and 3.21, respectively, $P=0.029$ ). The most frequently affected pathways in patients with primary refractoriness were signaling, transcription and tumor suppression, whereas methylation and splicing pathways were mainly implicated in relapsed patients. New therapeutic targets, either by an approved drug or within clinical trials, were not identified in any of the cases of refractoriness (zero of ten); however, eight potential new targets were found in five relapsed patients (five of 13, $P=0.027$ ): one IDH2, three SF3B1, two KRAS, one KIT and one JAK2. Sixty-five percent of all variants detected at diagnosis were not detected at complete response. Specifically, $100 \%$ of variants in EZH2, RUNX1, VHL, FLT3, ETV6, U2AF1, PHF6 and SF3B1 disappeared at complete response, indicating their potential use as markers to evaluate minimal residual disease for follow-up of AML. Molecular follow-up using a custom NGS myeloid panel of 32 genes in the post-treatment evaluation of AML can help in the stratification of prognostic risk, the selection of minimal residual disease markers to monitor the response to treatment and guide post-remission strategies targeting AML, and the selection of new drugs for leukemia relapse.

\section{Introduction}

Approximately $20-30 \%$ of all patients with acute myeloid leukemia (AML) show primary refractoriness to induction therapy without achieving complete remission (CR) and approximately $50 \%$ will relapse. ${ }^{1}$ Both primary refractoriness and relapse are therapeutic failures associated with adverse prognosis, with cure rates no higher than $10 \% .^{2-5}$

AML is often an oligoclonal disease at its origin, because tumor clones with diverse genetic identity are present within the same patient in greater or lesser representation. In the last decade, much progress has been made in our understanding of tumor purity and the representation of clonal and sub-clonal mutations, particularly in the role played by sub-clones in the clonal architecture in AML. ${ }^{6}$ In this context, the clonal architecture can be driven not only by a single predominant clone, but also in some
Ferrata Storti Foundation

Haematologica 2021

Volume 106(9):2325-2333

\section{Correspondence:}

ROSA AYALA

rosam.ayala@salud.madrid.org/rayala@ucm.es

Received: April 4, 2020.

Accepted: July 20, 2020.

Pre-published: July 30, 2020.

https://doi.org/10.3324/haematol.2020.254623

(C)2021 Ferrata Storti Foundation

Material published in Haematologica is covered by copyright. All rights are reserved to the Ferrata Storti Foundation. Use of published material is allowed under the following terms and conditions:

https://creativecommons.org/licenses/by-nc/4.0/legalcode. Copies of published material are allowed for personal or internal use. Sharing published material for non-commercial purposes is subject to the following conditions: https://creativecommons.org/licenses/by-nc/4.0/legalcode, sect. 3. Reproducing and sharing published material for commercial purposes is not allowed without permission in writing from the publisher. 
cases by several leukemic clones participating in the leukemic process, ${ }^{7}$ and even by genetically distinct clones segregating or combining, providing more tumor diversity., Consequently, the predominating clone at diagnosis may differ from the clone predominating in states of relapse or refractoriness. ${ }^{5}$

Previous reports on the origin and evolution of genomic mutations in AML suggest that the majority are random events that arose in hematopoietic stem/progenitor cells before they acquired the driver mutation. Patients with clonal hematopoiesis frequently present with mutations in the genes TET2, RUNX1 and EZH2, whereas patients without clonal hematopoiesis are associated with mutations in NPM1 and FLT3 ${ }^{10}$ Furthermore, one-third of all patients with myelodysplastic syndromes (MDS) evolve to AML through a process of clonal evolution involving mutations in several genes including NPM1, RUNX1, TP53 and NRAS. ${ }^{11}$

The reappearance of leukemic disease after relapse can be through several distinct mechanisms: i) the founding clone acquires new mutations, expands and emerges as the predominant clone at relapse; ii) a non-founding clone or subclone resists chemotherapy, acquires new mutations, expands and becomes the predominant clone in relapse; iii) an ancestral, pre-diagnostic clone evolves and emerges as the major clone at relapse; and iv) the treatment triggers the appearance of a new clone, not previously present, and generates a second pathology (not a relapse per se)..$^{12,13}$

In a very recent study examining a cohort of adult patients with AML with NPM1 mutated at diagnosis, common cancer pathways such as MAPK and WNT were found to be enriched in relapsed samples with loss of the NPM1 mutation, whereas MYC and SCF-KIT signaling pathways were enriched in relapsed samples with persistent NPM1 mutation. ${ }^{14}$ Similarly, in an examination of the genetic mechanisms of primary chemotherapy resistance in pediatric AML, mutations in FRMD8, DHX32, PIK3R1, SHANK3, MKLN1, WT1 and TP53 were maintained or even enriched in refractory disease with respect to diagnosis, and mutations in FLT3, PTPN11 and NRAS genes were eradicated. ${ }^{15}$

In the present study, we investigated the clinical impact of the molecular evolution of AML in patients after standard induction treatment. We performed genetic mutational studies along the follow-up in refractory and relapsed AML using targeted next-generation sequencing (NGS) with a 32-gene panel. This approach involved a complete analysis of paired samples at the time of diagnosis versus refractoriness and versus relapse from 23 patients who failed induction chemotherapy and/or who relapsed after CR.

\section{Methods}

\section{Patients}

The NGS-based mutational dynamics study was performed in a cohort of 23 AML patients with therapeutic failure, refractory to induction treatment $(n=8)$, relapsed after reaching $C R(n=13)$, and first refractory then relapsed $(n=2)$, diagnosed between 2007 and 2015 in the Hospital 12 de Octubre, Madrid. Patients were selected from a previous sequencing study at diagnosis $(n=190) .{ }^{16,17}$ The study evaluated 91 samples in total from the 23 cases at different time points: diagnosis $(n=23), C R(n=31)$, partial remission $(n=3)$, primary refractoriness $(n=13)$, second-line refractoriness $(n=4)$ and relapse $(n=17)$. The median age at diagnosis was 59 ( range, 24-77) years and patients were treated with cytarabine and idarubicin $(3+7$ scheme, $\mathrm{n}=21$ ) or with or in FLUGAZA clinical trial (azacytidine arm, $\mathrm{n}=2$ ) as induction treatment according to PETHEMA (Programa Español de Tratamientos en Hematologia) protocols. Other clinical characteristics are summarized in Table 1 . The study was conducted according to the Spanish law 14/2007 on biomedical research and was approved by the research ethics board of each participating institution. All patients provided informed consent.

\section{Mutational profile workflow}

DNA was extracted using Maxwell ${ }^{\circledR} 16 \mathrm{MDx}$ (Promega Biotech Iberica SL, Madrid, Spain) and quantified on a Qubit ${ }^{\circledR} 2.0$ Fluorometer (Invitrogen, Thermo Fisher Scientific Inc., Waltham, $\mathrm{MA})$. The sequencing workflow was done with a custom NGS myeloid panel of 32 genes frequently mutated in myeloid diseases (Online Supplementary Table S1). In addition, the detection and quantification of mutated NPM1 sequences was performed by allele specific quantitative polymerase chain reaction (qPCR), as previously described, ${ }^{17}$ using RNA as biological sample and $A B L 1$ as the expression reference gene for normalization. ${ }^{18}$ Internal tandem duplications in FLT3 were detected using GENSCAN. ${ }^{19}$ Fastq files were processed and genomic variants were detected using RUbioSeq3.8. ${ }^{20}$ which filtering and prioritization are detailed in Online Supplementary Figure S2.

\section{Statistical analysis}

All analyses were performed using the R environment (v3.4.4) for statistical computing. Fisher's exact test was used to determine differences between two categorical variables. The median follow-up time was 18.5 (range, 2.8-127) months.

Primary refractory AML was defined as the failure to achieve CR after the first cycle of induction treatment. Partial response AML was defined as having $5-19 \%$ blast cells in bone marrow with

Table 1. Clinical description of patients.

\begin{tabular}{lcc} 
Patients $(n=23)$ & Male & Female \\
Sex & $13(57 \%)$ & $10(43 \%)$ \\
Age at diagnosis & Years, median & 59 \\
& (range) & $(24-78)$ \\
\hline Blasts at diagnosis & $\%$, median & 70 \\
& (range) & $(8-06)$ \\
WBC at diagnosis & $10^{9}$ L, median & 8.7 \\
& (range) & $(1.2-145)$ \\
\hline AML type & De novo & $20(87 \%)$ \\
& t-AML & $3(13 \%)$ \\
Karyotype at diagnosis & Normal & $7(30 \%)$ \\
& Altered & $16(70 \%)$ \\
\hline Cytogenetics Risk Group & Low & $3(13 \%)$ \\
(ELN-2010) & Intermediate & $12(52 \%)$ \\
& High & $8(35 \%)$ \\
HSCT & Autologous & $2(9 \%)$ \\
& Allogenic & $9(39 \%)$ \\
& Not done & $12(52 \%)$ \\
\hline Induction therapy & $3+7$ scheme* & $21(91 \%)$ \\
& Azacytidine** & $2(9 \%)$ \\
\hline
\end{tabular}

The table represents clinical data of patients included in the NGS study.WBC: white blood cells; t-AML: secondary AML to other chemotherapy; HSCT: hematopoietic stem cell transplantation. ${ }^{*} 3+7$ regimen of chemotherapy: one or two induction cycles of cytarabine and idarubicin during seven and three days, respectively; and two or three consolidation cycles at high doses of cytarabine, twice a day for three alternate days followed by allogenic- or autologous-HSCT. * * Azacytidine scheme (azacytidine days 1 to 7 ). 
$>50 \%$ decrease after one cycle of induction treatment; for statistical evaluation a partial remission sample was considered as a refractory sample. ${ }^{21}$ Relapse AML was defined as the recurrence of disease after $\mathrm{CR}$, provided that it was detected in $\geq 5 \%$ blasts in the bone marrow or peripheral blood. The classification of clonal or subclonal mutation was derived from the variant allele frequency (VAF), which provides information about how many cells in a sample carry a particular variant. The VAF is defined as the ratio of sequence reads carrying the mutation to the total number of reads at a specific nucleotide position. In this study, $\mathrm{VAF} \geq 10 \%$ discriminates a clonal mutation and $\mathrm{VAF}<10 \%$ a subclonal mutation. Accordingly, the predominant clone is the one with a higher VAF. Also, additional molecular abnormalities (AMA) in patients with refractory or relapsed disease were defined as new mutations not present at diagnosis.

\section{Results}

Patient cohorts and clinical-biological characteristics

We observed a dynamic mutational profile along the course of AML evaluation, both for samples from patients refractory to induction treatment (patients 1 to 10; Figure $1 \mathrm{~A}$ and $B$ ) and from patients who relapsed after reaching CR (patients 9 to 23; Figure $1 \mathrm{~A}$ to $\mathrm{C}$ ). A detailed description of biological and clinical events, and specific treatments of the patients is reported in the Online Supplementary Appendix.

\section{Mutational landscape at diagnosis and treatment failure}

Overall, 71 non-recurrent somatic variants were detected with a median coverage of 1,044 (range, 20-6,123) and a median VAF of $36 \%$ (range, 1-95\%) (Online Supplementary Table S2). Fifty-one variants $(71.8 \%)$ were single nucleotide variants (SNV) and $20(28.2 \%)$ were small insertions $(n=15)$ or deletions $(n=5)$. Fifty missense variants were identified, in addition to one stop-gain, one in-frame deletion, four frameshift deletions, 14 frameshift insertions and one nonframeshift insertion.

At diagnosis $(n=23), 57$ variants were detected with a median of twp (range, $0-5$ ) variants/sample. We did not find any mutations in three patients, either at the beginning of

A
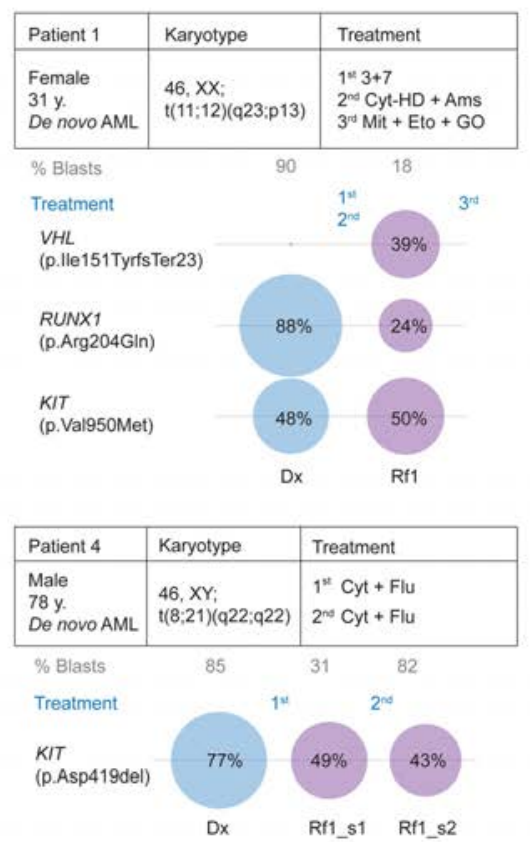

\begin{tabular}{|c|c|c|}
\hline Patient 7 & Karyotype & Treatment \\
\hline $\begin{array}{l}\text { Female } \\
36 \text { y. } \\
\text { De novo AML }\end{array}$ & $\begin{array}{l}45, X X \\
\text { dup }(19),-16\end{array}$ & $1=3+7$ \\
\hline \multicolumn{2}{|l|}{$\%$ Blasts } & 8 \\
\hline \multicolumn{2}{|l|}{ Treatment } & $1^{\mathrm{u}}$ \\
\hline \multicolumn{2}{|c|}{ No mutations detected } & $0^{+} \quad \beta^{2}$ \\
\hline
\end{tabular}
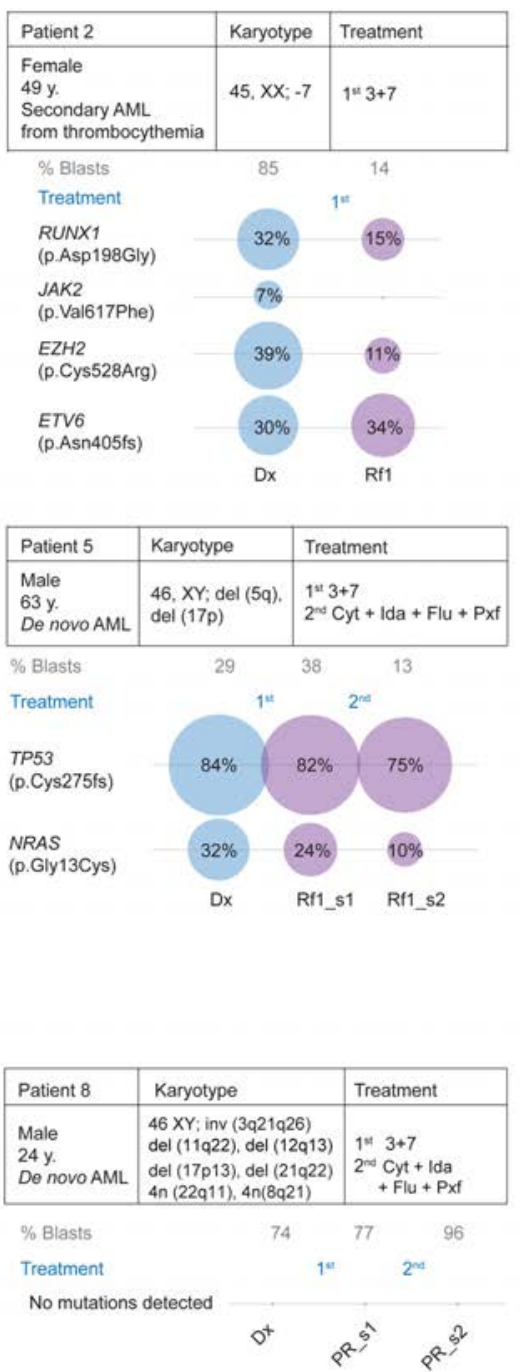

Figure 1A. Legend on page 2329.
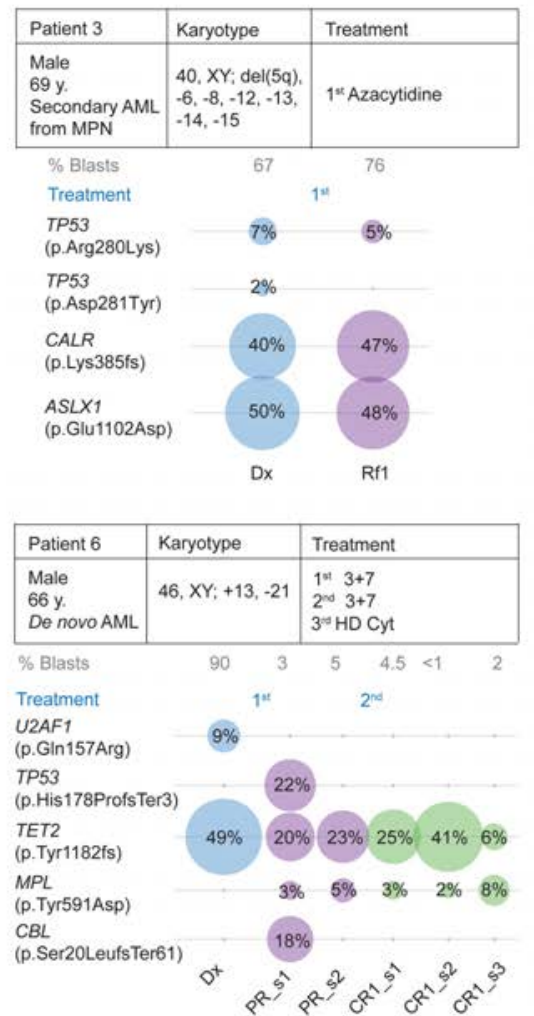

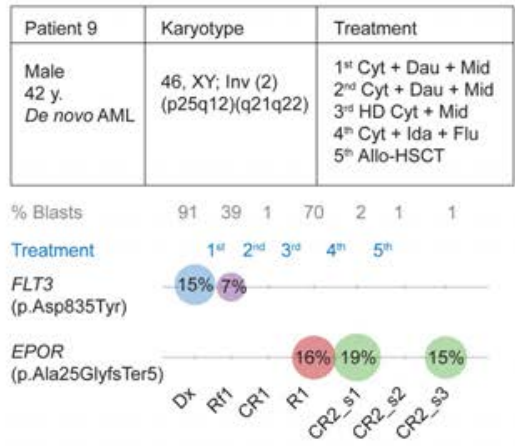


B
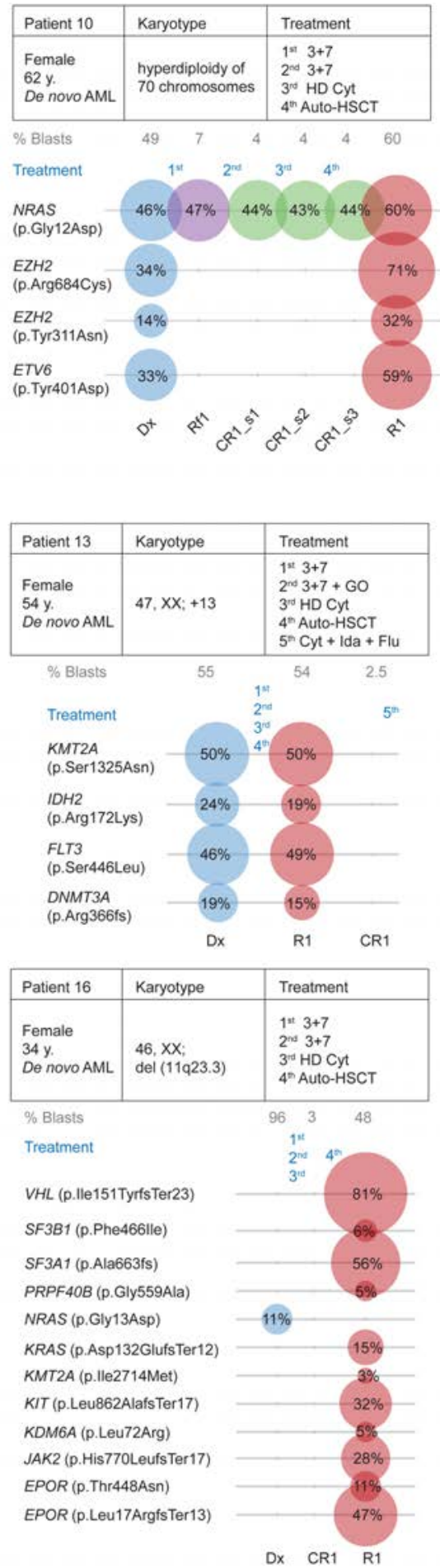

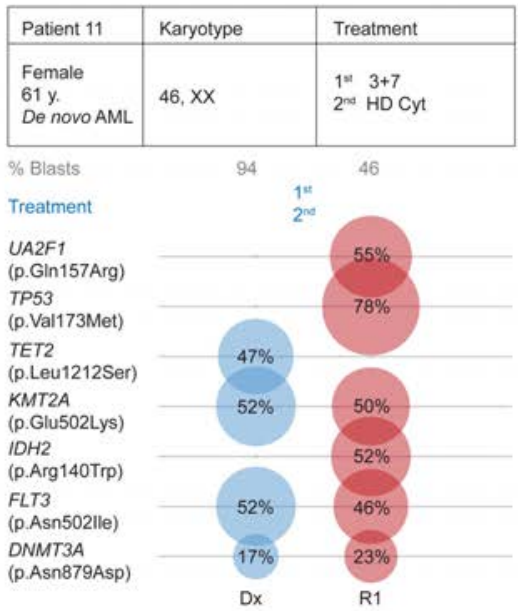

\begin{tabular}{|c|c|c|}
\hline Patient 12 & Karyotype & Treatment \\
\hline $\begin{array}{l}\text { Male } \\
28 \mathrm{y} \text {. } \\
\text { De novo AML }\end{array}$ & $\begin{array}{l}\text { 46, XY; Inv(16) } \\
\text { (p13;q22), } \\
\text { positive } \\
\text { CBF/MYH11 }\end{array}$ & $\begin{array}{l}1^{\text {st }} 3+7 \\
2^{\text {sed }} \mathrm{HD} \mathrm{Cyt} \mathrm{+} \mathrm{Ida} \\
3^{\text {st }} \mathrm{HD} \text { Cyt + Ams } \\
4^{\text {s }} \text { Auto-HSCT }\end{array}$ \\
\hline
\end{tabular}
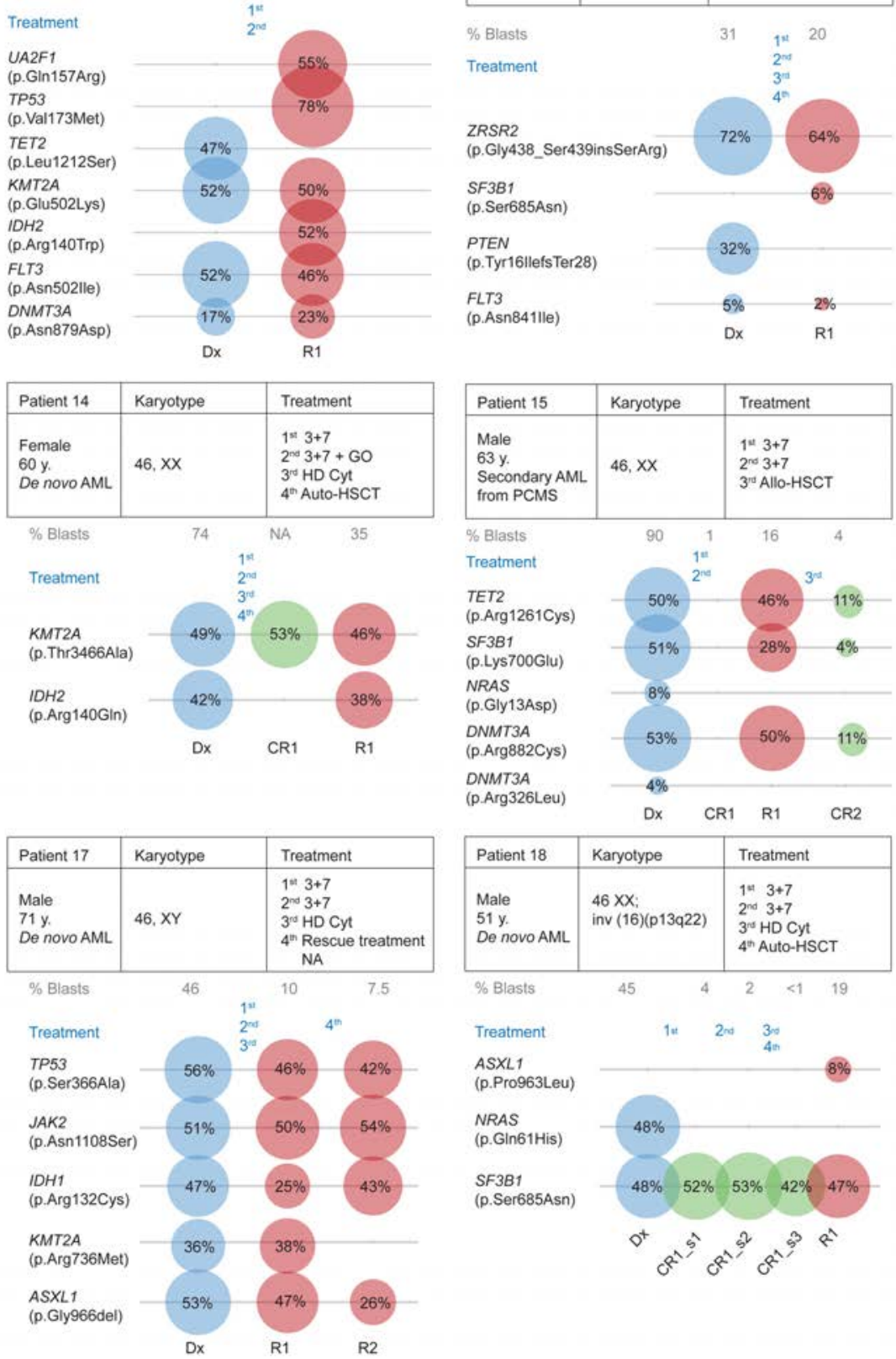

\begin{tabular}{|c|c|c|}
\hline Patient 18 & Karyotype & Treatment \\
\hline $\begin{array}{l}\text { Male } \\
51 \text { y. } \\
\text { De novo AML. }\end{array}$ & $\begin{array}{l}46 X X \\
\text { inv }(16)(p 13 q 22)\end{array}$ & $\begin{array}{l}1^{\text {nt }} 3+7 \\
2^{\text {nd }} 3+7 \\
3^{\text {ss }} \mathrm{HD} \mathrm{Cyt} \\
4^{\text {th }} \text { Auto-HSCT }\end{array}$ \\
\hline$\%$ Blasts & 45 & $2<1$ \\
\hline
\end{tabular}

Treatment

ASXL1

(p.Pro963Leu)

NRAS

(p.Gln61His)

SF3B1

(p.Ser685Asn)

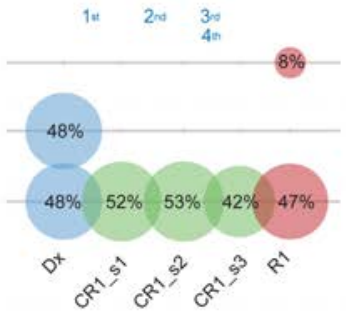

Figure 1B. Legend on following page.

the disease or at any other time during evaluation; furthermore three of 23 cases presented with an altered karyotype at diagnosis. In the analysis of the samples of treatment refractoriness, we detected a median of one (range, 0-4) variant/sample in 20 samples evaluated, with three samples having no mutations. At $\mathrm{CR}$, we detected a median of one (range, 0-4) variant/sample in 31 samples evaluated, with 11 samples having no mutations. At relapse, we detected a median of three (range, $0-11$ ) variants/sample in 17 samples evaluated, one of which did not have a mutation (Online
Supplementary Figure S1).

Differences in genomic features between primary refractoriness and relapse at diagnosis

Cases of primary refractoriness were associated with high-risk cytogenetics (ELN-2010 criteria) ${ }^{22}$ (six of nine), whereas relapsed cases were related to intermediate-risk cytogenetics ELN-201022 (nine of $14, P=0.085$ ). Also, the cases of primary refractoriness were associated with a lower number of variants at diagnosis (median 1.67) than leukemia relapses cases, with a median of 3.21 variants $(P=0.029)$. 
C
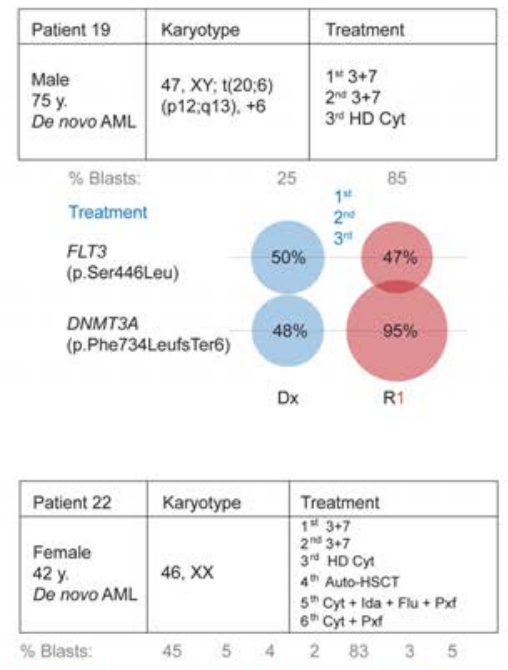

Treatment

PHF6

(p. Gly275Glu)

CBL
p.Met119lle
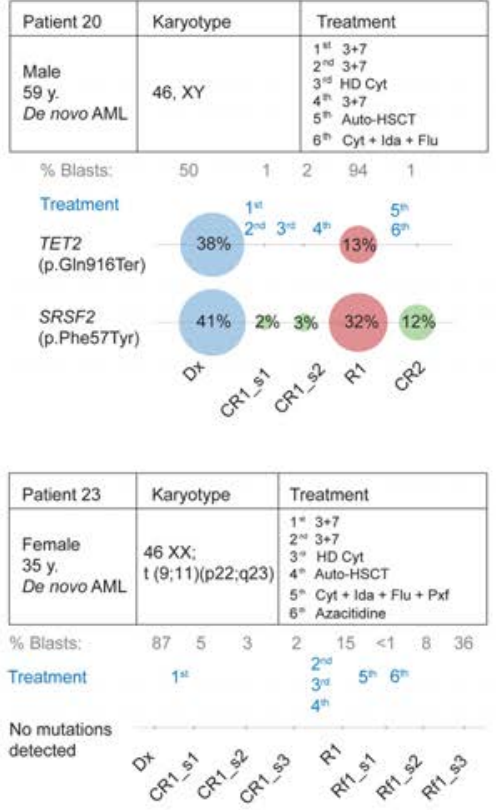

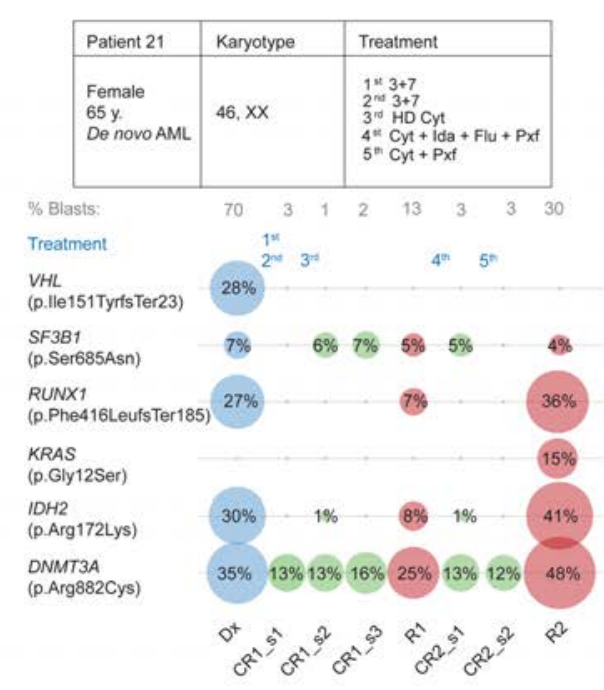

(⿸丆口

Figure 1. Mutational features of patients. (A) The graphs show the mutational profile after induction treatment in primary refractory cases (patients 1 to 8 ), and patients who present with both primary refractory disease and relapse after complete remission (CR) (patient 9). (B) The graphs show the mutational profile after induction treatment in patients who present with both primary refractory disease and relapse after CR (patient 10), and patients who relapse after induction treatment (patients 11 to 18). (C) The graphs show the mutational profile after induction treatment in relapse cases (patients 19 to 23 ). The balls represent the presence of mutations, with their size representing the percentage variant allele frequency $(\% \mathrm{VAF})$ and the color representing the moment of evaluation $(\mathrm{dx}=\mathrm{blue}$, $\mathrm{CR}=\mathrm{green}$, $\mathrm{PR}$ and $\mathrm{Rf}=$ purple, $\mathrm{R}=\mathrm{red}$ ). Sex, age and type of acute myeloid leukemia are indicated in the individual table, as well as karyotype and treatment administrated. The percentage of blasts is indicated above the mutation, and the time-frame of treatment cycles is indicated in the same way. y: years; HD-Cyt: high-dose cytosine; Ams: amsacrine; Mit: mitoxantrone; Eto: etoposide, GO: gemtuzumab; Clo: clofarabine; Flu: fludarabine; Ida: idarubicine; Pxf: plerixafor; Dau: daunorubicin; Mid. Midostaurin; allo-HSCT: allogeneic hematopoietic stem cell transplant; auto-HSCT: autologuos hematopoietic stem cell transplant; NA: not available. Dx=diagnosis, CR=complete remission, $\mathrm{PR}=$ partial remission and $\mathrm{R}=$ =refractoriness and $\mathrm{R}=$ relapse. In the case of several samples at time of evaluation, the samples were labeled sequentially (s1=sample 1, s2=sample 2, s3 = sample 3 and successively). Also, the mutated gene and variant (protein coding) and the exact \% of VAF is indicated.

At diagnosis, we observed that in the group of patients who had shown primary refractoriness, the most frequently mutated genes were those associated with tyrosine kinases (KIT, NRAS, CBL, RUNX1) and TP53. By contrast, genes related to epigenetic regulation (DNMT3A, IDH1/2, KMT2A) and SF3B1 were more frequently mutated in the group of patients who subsequently relapsed (Online Supplementary Table S2).

\section{Clonal evolution is involved with dynamics of variant allele frequency}

Almost $68 \%$ (67.7\%) of the variants in the follow-up samples evaluated after induction cycles were the same as those detected at diagnosis (Table 2): TP53 ( $n=3 / 4), N R A S(n=3 / 3)$, $\operatorname{KIT}(\mathrm{n}=3 / 3), \operatorname{CBL}(\mathrm{n}=3 / 3), \operatorname{RUNX} 1(\mathrm{n}=2 / 2), \operatorname{TET} 2(\mathrm{n}=2 / 2)$, ASXL1 ( $\mathrm{n}=1 / 1)$, CALR ( $\mathrm{n}=1 / 1)$, EZH2 ( $\mathrm{n}=1 / 3)$, FLT3-SNV $(n=1 / 1)$ and ETV6 $(n=1 / 2)$. We also detected the following newly acquired mutations at treatment refractoriness: $M P L$ $(\mathrm{n}=2)$, CBL $(\mathrm{n}=1), \mathrm{TP} 53(\mathrm{n}=1)$, and VHL $(\mathrm{n}=1)$. Notably, several variants were detected at diagnosis and disappeared at refractoriness (32.3\%): PHF6 ( $\mathrm{n}=3 / 3)$, U2AF1 $(\mathrm{n}=2 / 2), J A K 2$ $(\mathrm{n}=1 / 1)$, EZH2 $(\mathrm{n}=2 / 3)$, ETV6 $(\mathrm{n}=1 / 2)$ and TP53 $(\mathrm{n}=1 / 4)$.

When we analyzed paired relapsed and diagnosis samples (Table 2), the variants that were maintained (80.7\%) in relapsed samples were located in the following genes: DNMT3A $(\mathrm{n}=6 / 7)$, SF3B1 $(\mathrm{n}=3 / 3)$, KMT2A $(\mathrm{n}=4 / 5)$, TP53 $(\mathrm{n}=2 / 2)$, IDH2 $(\mathrm{n}=4 / 4)$, FLT3-SNV $(\mathrm{n}=4 / 5)$, TET2 $(\mathrm{n}=3 / 4)$,
ASXL1 (n=3/3), JAK2 (n=2/2), RUNX1 (n=2/2), EZH2 $(\mathrm{n}=2 / 2), \operatorname{IDH} 1(\mathrm{n}=2 / 2)$, CBL $(\mathrm{n}=1 / 1), \operatorname{NRAS}(\mathrm{n}=1 / 5)$, ETV6 $(\mathrm{n}=1 / 1)$, PHF6 $(\mathrm{n}=1 / 1)$, SRSF2 $(\mathrm{n}=1 / 1)$ and ZRSR2 $(\mathrm{n}=1 / 1)$. In addition, we detected 18 variants that were newly acquired during the progression: SF3B1 ( $\mathrm{n}=3), \operatorname{EPOR}(\mathrm{n}=3), K R A S$ $(\mathrm{n}=2), I D H 2(\mathrm{n}=1), \operatorname{KMD} 6 A(\mathrm{n}=1), \operatorname{KMT} 2 A(\mathrm{n}=1), \operatorname{KIT}(\mathrm{n}=1)$, PRPF4OB ( $\mathrm{n}=1)$, SF3A1 ( $\mathrm{n}=1)$, U2AF1 ( $\mathrm{n}=1), J A K 2(\mathrm{n}=1), V H L$ $(\mathrm{n}=1)$ and TP53 $(\mathrm{n}=1)$. By contrast, the variants detected at diagnosis but that disappeared $(19.3 \%)$ in relapsed samples were located in genes: NRAS $(\mathrm{n}=4 / 5), D N M T 3 A(\mathrm{n}=1 / 8)$, KMT2A $(\mathrm{n}=1 / 5)$ FLT3-SNV $(\mathrm{n}=1 / 5)$, TET2 $(\mathrm{n}=1 / 4)$, VHL $(\mathrm{n}=2 / 2)$ and PTEN $(n=1 / 1)$. These latter clones could be sensitive to treatment.

We observed a decreased mutational load of $8.1 \%$ in samples from treatment refractory patients versus diagnosis samples (Figure 2A). While, an increased mutational load of $3.74 \%$ in the relapsed versus diagnosis samples (Figure $2 \mathrm{~B}$ ).

\section{Molecular findings in complete remission of leukemia}

Regarding CR evaluation, we detected 81 variants in the study of paired CR and diagnosis samples (Table 2). The variants detected at diagnosis and maintained in CR (34.6\%) were located in TET2 ( $\mathrm{n}=4 / 8)$, DNMT3A $(\mathrm{n}=6 / 10)$, ASXL1 $(\mathrm{n}=3 / 3), \operatorname{NRAS}(\mathrm{n}=3 / 9), \operatorname{KMT} 2 A(\mathrm{n}=1 / 2), \operatorname{SF3B1}(\mathrm{n}=3 / 6)$, IDH2 $(\mathrm{n}=2 / 7)$, SRSF2 $(\mathrm{n}=3 / 3)$ and $C B L(\mathrm{n}=2 / 2)$. In addition, the variants that disappeared in CR $(65.4 \%)$ were located in IDH2 $(\mathrm{n}=5 / 7)$, NRAS $(\mathrm{n}=6 / 9)$, DNMT3A $(\mathrm{n}=4 / 10)$, TET2 
Table 2. Variants detected in refractoriness, complete remission and relapse versus diagnosis.

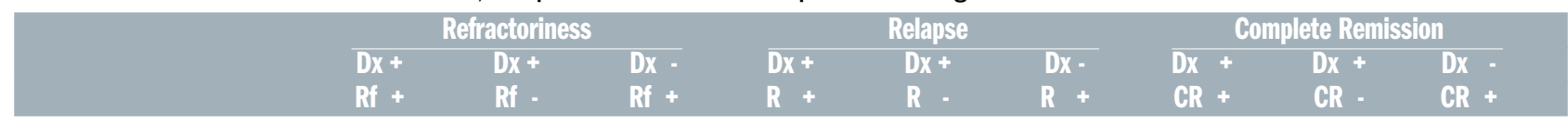

\begin{tabular}{|c|c|c|c|c|c|c|c|c|c|}
\hline ASXL1 & 1 & & & 3 & & & 3 & & \\
\hline CALR & 1 & & & & & & & & \\
\hline$C B L$ & 3 & & 1 & 1 & & & 2 & & \\
\hline DNMT3A & & & & 6 & 1 & & 6 & 4 & \\
\hline EPOR & & & & & & 3 & & & 2 \\
\hline ETV6 & 1 & 1 & & 1 & & & & 3 & \\
\hline EZH2 & 1 & 2 & & 2 & & & & 6 & \\
\hline FLT3-SNV & 1 & & & 4 & 1 & & & 6 & \\
\hline IDHI & & & & 2 & & & & & \\
\hline IDH2 & & & & 4 & & 1 & 2 & 5 & \\
\hline JAK2 & & 1 & & 2 & & 1 & & & \\
\hline KMD6A & & & & & & 1 & & & \\
\hline KIT & 3 & & & & & 1 & & & \\
\hline KMT2A & & & & 4 & 1 & 1 & 1 & 1 & \\
\hline$K R A S$ & & & & & & 2 & & & \\
\hline MPL & & & 2 & & & & & & 3 \\
\hline$N R A S$ & 3 & & & 1 & 3 & & 3 & 6 & \\
\hline PHF6 & & 3 & & 1 & & & & 2 & \\
\hline PRPF40B & & & & & & 1 & & & \\
\hline PTEN & & & & & 1 & & & & \\
\hline RUNXI & 2 & & & 2 & & & & 5 & \\
\hline SF3Al & & & & & & 1 & & & \\
\hline SF3B1 & & & & 3 & & 3 & 4 & 3 & \\
\hline SRSF2 & & & & 1 & & & 3 & & \\
\hline TET2 & 2 & & & 2 & 1 & & 4 & 4 & \\
\hline TP53 & 3 & 1 & 1 & 2 & & 1 & & & \\
\hline U2AF1 & & 2 & & & & 1 & & 3 & \\
\hline VHL & & & 1 & & 2 & 1 & & 5 & \\
\hline ZRSR2 & & & & 1 & & & & & \\
\hline$N$ & 21 & 10 & 5 & 42 & 10 & 18 & 28 & 53 & 5 \\
\hline
\end{tabular}

The table lists the number of samples with allelic variants detected in the different genes included in the study.The table specifies the samples studied in primary refractoriness, relapse and complete remission. The last row indicates the number of samples that are present at diagnosis $(\mathrm{Dx}+)$, at refractoriness $(\mathrm{Rf}+)$, at relapse $(\mathrm{R}+)$ or at complete remission $(\mathrm{CR}+)$.Variants that are present in the diagnosis $(\mathrm{Dx}+)$ but absent in refractoriness $(\mathrm{Rf}-)$ or relapse ( $\mathrm{R}-)$ or in complete remission (CR-). Thus, there are variants absent in the diagnosis $(\mathrm{Dx}-)$ but are present in refractoriness $(\mathrm{Rf}+)$ or relapse $(\mathrm{R}+)$ or complete remission $(\mathrm{CR}+)$.

$(\mathrm{n}=4 / 8)$, EZH2 $(\mathrm{n}=6 / 6)$, RUNX1 $(\mathrm{n}=5 / 5), V H L(\mathrm{n}=5 / 5)$, FLT3 $(\mathrm{n}=6 / 6)$, SF3B1 $(\mathrm{n}=4 / 6)$, ETV6 $(\mathrm{n}=3 / 3)$, U2AF1 $(\mathrm{n}=3 / 3)$, PHF6 $(\mathrm{n}=2 / 2)$, y $\operatorname{KMT} 2 A(\mathrm{n}=1 / 2)$. Also, five variants arose de novo in DNMT3A ( $\mathrm{n}=2)$ and MPL ( $\mathrm{n}=3)$. These results provide potential markers that could be used to detect minimal residual disease (MRD) in our series, including EZH2, RUNX1, VHL, FLT3, ETV6, U2AF1, PHF6 and SF3B1, as these variants disappear in CR.

\section{Branching clonal evolution is predominant in acute myeloid leukemia}

Analysis of the molecular dynamics of the clones according to the VAF identified three patients who showed a change in the predominant clone from diagnosis to primary refractoriness: clones characterized by mutations in VHL (patient 1), ETV6 (patient 2) and TP53 (patient 6) became the predominant clones at refractoriness (Figure 1A). Likewise, four relapsed patients showed changes in the predominant clone from diagnosis, characterized by mutations in EPOR (patient 9), TP53 (patient 11), VHL (patient 16) and PHF6 (patient 22) (Figure 1A to C). In addition, clonal evolution was observed in 12 patients. A linear clonal evolution model was identified in four patients in primary refractoriness and two in relapse. By contrast, a branching clonal evolution model was identified in two patients in primary refractoriness and in six patients in relapse.

Subclonal mutations (VAF $<10 \%$ ) were detected at diagnosis in signaling pathway genes (JAK2, FLT3, NRAS) and in splicing genes (U2AF1, SF3B1). Of these, JAK2, NRAS and $U 2 A F 1$ variants disappeared in the treatment failure samples, whereas FLT3 and SF3B1 variants persisted. Also, we detected variants in TP53 and PHF6 (tumor suppressor genes), but only the $P H F 6$ variant became the predominant clone in a relapsed sample (VAF=83\%). Two TP53-subclonal mutations were detected at diagnosis in the same patient, but only one of them was maintained at a similar frequency in the refractory sample. 
A

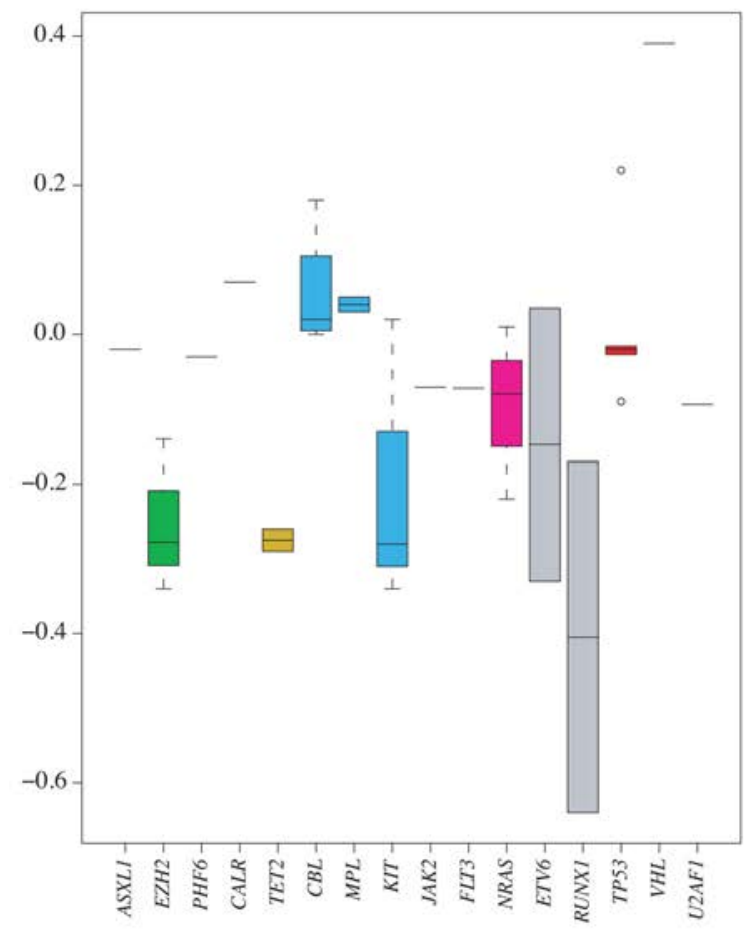

B

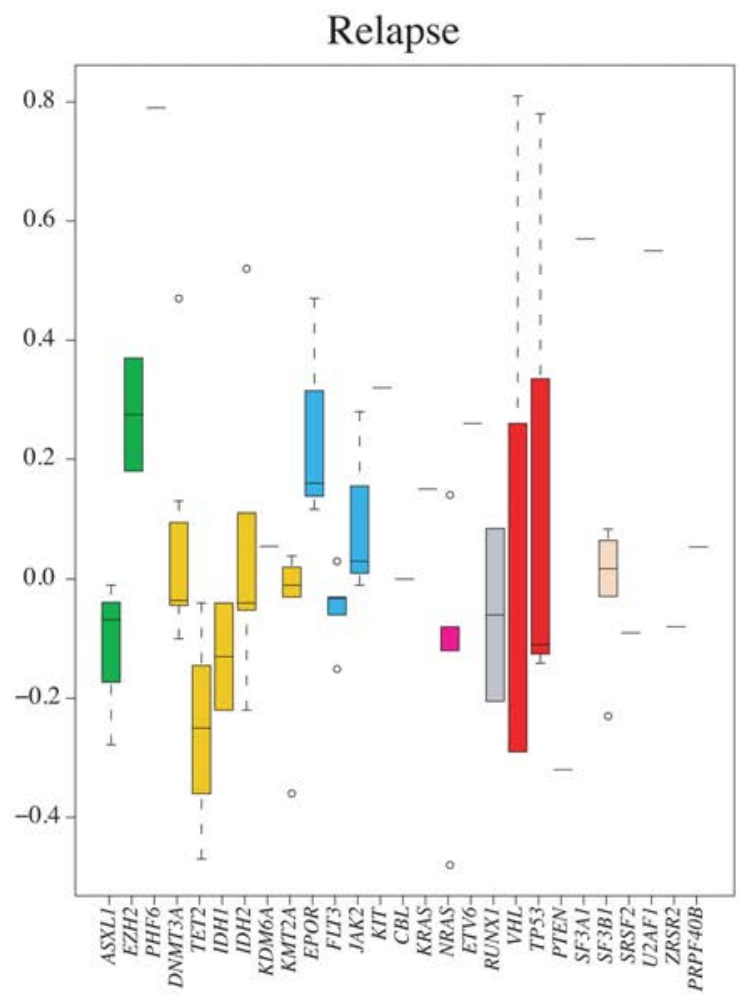

Figure 2. Variation of the allelic frequency of the variants. Box plots representing the increase or decrease of allelic frequencies detected in genes where variants have been detected at refractoriness. (A) and at relapse (B). The genes are grouped according to metabolic pathways, with different colors representing: transcriptional regulator genes (ASXL1, EZH2 and PHF6) in green, CALR in black, epigenetic regulator genes (DNMT3A, TET2, IDH1, IDH2, KDM6A and KMT2A) in yellow, splicing genes (SF1, SF3A1, SF3B1, SRSF2, U2AF1, ZRSR2 and PRPF4OB) in brown, cytokine signaling and JAK/STAT pathway genes (EPOR, FLT3, JAK2, KIT, SH2B3, MPL and CBL) in blue, GTPase activity genes (HRAS, KRAS and NRAS) in pink, transcription factors genes (ETV6 and RUNX1) in grey and tumor suppressor genes (VHL, TP53 and PTEN) are represented in red.

\section{Clonal evolution is not associated with a worse outcome}

Conventional molecular alterations detected at diagnosis in ten patients were lost both at refractoriness $(n=3)$ and leukemia relapse $(n=7)$. AMA were identified in eight patients, who all achieved CR (eight of 17 cases with any $\mathrm{CR}$ vs. zero of six cases with no CR; $P=0.037)$. Median overall survival was 77.4 (range, 21.5-133.3) months for the group with AMA features versus 11.8 (range, 1.2-22.4) months for the group without AMA features $(P=0.083$, Online Supplementary Figure 3SA). Clonal evolution detected with AMA identified patients with a trend for a better prognosis for disease-free survival (median disease-free survival was 22.1 vs. 10.8 months; $P=0.065$, Online Supplementary Figure 3SB); however, neither loss of molecular abnormalities nor combined additional and lost molecular abnormalities had an impact on prognosis.

Accrual of AMA was mainly related to signaling pathway genes (five of eight cases cases with gain of mutations in the genes of the signaling pathway compared with only three of 15 cases without gain of mutations; $P=0.042$ ). Loss of AMA in relapsed samples was also mainly found for signaling pathway genes (seven of ten cases with loss of AMAs present in signaling pathways genes compared with one of 13 cases without loss of AMA; $P=0.002$ ).

Other variables with a trend for an association with cases who achieved CR were normal karyotype (six of 17 cases with any $\mathrm{CR}$ vs. zero of six cases with no CR; $P=0.091)$ and cytogenetic risk (only six of ten high-risk, but 11 of 13 intermediate- or low-risk achieved CR; $P=0.183$ ).
New therapeutic targets, either by an approved drug or within clinical trials, were not identified in cases of refractoriness; however, eight potential new targets were found in five relapsed cases (zer of ten refractoriness cases $v s$. five of 13 relapse cases, $P=0.027)$ : one $I D H 2$, three $S F 3 B 1$, two $K R A S$, one KIT and one JAK2.

\section{Discussion}

Patients with AML who show primary resistance to induction treatment or leukemic relapse have a dismal prognosis. Our study identifies differences in the mutational landscape between primary refractory and relapsed AML. In this line, we report the usefulness of monitoring different leukemic clones, and particularly detecting the appearance of new clones, using an NGS-targeted panel in post-treatment AML, allowing us to: i) stratify patients into prognostic risk groups; ii) select $M R D$ marker/s to monitor response to treatment; and ii) define targeted post-remission strategies including the selection of new drugs for leukemia relapse.

The genetic follow-up of leukemic clones was performed using NGS technology with a high coverage of the variants $(>1,000 \times)$, allowing the detection of sub-clones with a high sensitivity $(<3 \%)$ in a cohort of 23 patients with AML, and with almost 100 samples evaluated. The NGS technology also allowed us to estimate the mutational load based on the VAF level, and to, therefore, infer the clonal architecture of the tumor and the model of clonal evolution. ${ }^{6}$ We confirmed the high clonal heterogeneity associated with this disease and the mutational profile associated with treatment refrac- 
toriness and relapse.

The genes most frequently mutated at diagnosis in patients showing subsequent primary refractoriness were CBL, KIT, NRAS, RUNX1 and TP53, and those more frequently mutated in relapsed patients were DNMT3A, $I D H 1 / 2, K M T 2 A$ and SF3B1. Thus signaling, transcription and tumor suppression pathways were the more affected biological categories at diagnosis in the treatment refractory group, whereas methylation and splicing were the pathways most affected at diagnosis in the relapsed group. This perhaps indicates that methylation and splicing are rescue pathways used by leukemia cells to develop resistance to treatment. Our findings may lead to the development of a new focused therapeutic approach for patients belonging to the high-risk cytogenetics group at relapse but who have nevertheless achieved CR, because the oncologist could anticipate maintenance treatments based on drugs targeting methylation and splicing pathways, for example, hypomethylants or splicing inhibitors. Rescue pathways were not identified for cases of primary refractoriness, as clones remained stable and alterations were found in signaling and tumor suppressor genes, which are the most clinically relevant.

We identified new potential therapeutic targets at the leukemia relapse stage affecting $I D H 2, S F 3 B 1$, KRAS, KIT and JAK2 genes, all of which are targets for approved drugs or available within clinical trials. However, some identified variants in SF3B1 and FLT3 are categorized as variant of uncertain (or unknown) significance and for clinical decision making only pathogenic or probably pathogenic variants can be used. Supporting our findings, a recent study described de novo mutations in transcription factors, signaling, cohesin and splicing pathways at the time of leukemia relapse in the $t(8 ; 21)$ AML patient subgroup $;^{23}$ however, mutations detected at diagnosis in epigenetic regulators and genes involved in cell cycle control were stable or disappeared. ${ }^{23}$

We also detected an equal percentage of cases (32.3\%) where the dominant clone changed within the refractory group versus the leukemia relapsed group with evolution of the following genes: ETV6,VHL, EPOR, JAK2, TP53, and PHF6. Consequently, therapeutic approaches must be targeted specifically to these clones if they are detected at diagnosis.

The impact of subclonal mutations detected at diagnosis and their usefulness as MRD markers is not yet defined. In our AML series, $55 \%$ of subclones detected at diagnosis were lost in failure samples and the other $45 \%$ remained as subclones. Only the PHF6 mutation became the predominant clone in a failure sample, supporting the concept that treatment can result in subclonal eradication, but whether a resistance-mediating mutation determines the presence of a corresponding subclone from diagnosis could found a future relapse.

We observed an increased mutational load as a strong molecular feature of relapse, as previously established in other hematological malignancies. ${ }^{24}$ In addition, the authors of the aforementioned study suggest that the knowledge of the tumor burden is important in the identification of subclones, with the aim of targeted and specific therapies to eradicate them and to follow their evolution.

In an analysis of over 4,000 patients with newly diagnosed AML, several biological-clinical variables (age, performance status, white blood cell count, secondary disease, cytogenetic risk and NPM1/FLT3-ITD mutational status) were each strongly and independently associated with resistance $(P<0.001)$; however, their ability to predict resistance was only fair. ${ }^{25}$ Our study includes other molecular markers (AMA) that improve the prediction of failure to response to leukemia relapse treatment $(P=0.066)$, although it does not predict it for refractoriness $(P=$ not signifcant). This is likely justified by the fact that the refractory group was enriched with high-risk cytogenetics features at diagnosis, whereas the relapsed group was associated with a greater number of pathogenetic or likely pathogenetic variants at diagnosis. In contrast to other reported findings ${ }^{26} \mathrm{we}$ did not detect differences in the age of the patients between the group with persistence of these mutations and the group without these mutations.

We found persisting DNMT3A, TET2 and ASXL1 variants in CR, which have been previously reported ${ }^{26}$ and are related to clonal hematopoiesis of indeterminate potential (with oncogenic potential). ${ }^{27}$ Other mutations, such as those involving KMT2A, CBL and NRAS, could be associated with AML transformation from a prior clonal disease, as described by Bejar. ${ }^{28}$ These differ from reported mutations that were involved in leukemic hematopoiesis in NPM1, PHF6, SRSF2, RUNX1 and TP53, 27,29-36 although de novo cases predominated in our series.

To the best of our knowledge, we provide the first clinical evidence that clonal evolution defined as AMA is a feature associated with cases that achieve CR and that have a better prognosis for disease-free survival. A previous study in AML provided a contrary prediction, which in an analogous manner defined the clonal evolution as additional cytogenetic abnormalities, and observed a worse prognosis. ${ }^{37}$ Our prediction agrees with reported findings in NPM1mutated AML, ${ }^{14}$ whereby AML patients with clonal evolution $\left(N P M 1^{-}\right)$at relapse have a significantly longer remission duration than patients without clonal evolution $\left(N P M 1^{+}\right)$. The better prognosis associated with patients with new clones (AMA) might be due to the fact that the treatment has been effective in the basal clone.

Previous studies have shown an association between persisting clonal cytogenetic markers in first remission and an increased risk of relapse. ${ }^{80,39}$ Somatic mutations that activate signaling pathways (FLT3, KRAS, or NRAS) were usually cleared on day 30 , suggesting that subclones containing these mutations may be more sensitive to induction chemotherapy. ${ }^{2}$ Although new advances in induction treatment for AML have improved the rates of CR and overall survival, most patients ultimately relapse without effective post-remission therapy. ${ }^{40}$

The utility of clonal dynamics studies can be tested with new treatments such as FLT3, IDH1 and IDH2 inhibitors. The recommended study time to perform the monitoring of clonal evolution would be at diagnosis, at the end of the induction in $\mathrm{CR}$, and at the refractory or relapsed stages, with the main utility in those patients with AML who achieve CR or blast clearance. Although some conclusions obtained need to be validated in another wide series, the results of the relevance of clonal kinetics and its implications are robust.

Our results suggest that the monitoring of clonal evolution by genomic approaches can help to select post-remission strategies to target AML, and may improve prediction of clonal evolution and response of treatment.

\section{Disclosures}

No conflicts of interest to disclose. 


\section{Contributions}

EO collected samples, performed experiments, analyzed and interpreted data, and wrote the manuscript; IR analyzed and interpreted data; $L M, G C-T, X G, M G$, JS interpreted data; PM collected samples and clinical data; RA and JML designed and supervised research and experiments, analyzed and interpreted data, and wrote the manuscript; all authors prepared the report and approved the final version.

\section{Acknowledgments}

The study was conducted according to the Declaration of
Helsinki; the protocol was reviewed and approved by the Institutional Review Board/Independent Ethics Committe of the participating centers.

\section{Funding}

This study was supported by the Subdireccion General de Investigación Sanitaria (Instituto de Salud Carlos III, Spain) grants PI13/02387 and PI16/01530, and the CRIS against Cancer foundation, grant 2014/0120. ML holds a postdoctoral fellowship of the Spanish Ministry of Economy and Competitiveness (FPDI-201316409).

\section{References}

1. Kuehn EW, Walz G, Benzing T. Von hippellindau: a tumor suppressor links microtubules to ciliogenesis and cancer development. Cancer Res. 2007;67(10):4537-4540.

2. Klco JM, Miller CA, Griffith $M$, et al. Association between mutation clearance after induction therapy and outcomes in acute myeloid leukemia. JAMA. 2015; 314(8):811-822

3. Breems DA, Van Putten WL, Huijgens PC, et al. Prognostic index for adult patients with acute myeloid leukemia in first relapse. J Clin Oncol. 2005;23(9):1969-1978.

4. Pemmaraju N, Kantarjian H, Garcia-Manero $\mathrm{G}$, et al. Improving outcomes for patients with acute myeloid leukemia in first relapse: a single center experience. Am J Hematol. 2015;90(1):27-30

5. Bose P, Vachhani P, Cortes JE. Treatment of relapsed/refractory acute myeloid leukemia. Curr Treat Options Oncol. 2017; 18(3):17.

6. Vosberg S, Greif PA. Clonal evolution of acute myeloid leukemia from diagnosis to relapse. Genes Chromosomes Cancer. 2019;58(12):839-849.

7. De S, Ganesan S. Looking beyond drivers and passengers in cancer genome sequencing data. Ann Oncol. 2017;28(5):938-945.

8. Barber LJ, Davies MN, Gerlinger $M$. Dissecting cancer evolution at the macroheterogeneity and micro-heterogeneity scale. Curr Opin Genet Dev. 2015;30:1-6.

9. Gerlinger M, Rowan AJ, Horswell S, et al. Intratumor heterogeneity and branched evolution revealed by multiregion sequencing. N Engl J Med. 2012;366(10):883-892.

10. Welch JS, Ley TJ, Link DC, et al. The origin and evolution of mutations in acute myeloid leukemia. Cell. 2012;150(2):264-278.

11. Genovese G, Kahler AK, Handsaker RE, et al. Clonal hematopoiesis and blood-cancer risk inferred from blood DNA sequence. N Engl J Med. 2014;371(26):2477-2487.

12. Grimwade D, Ivey A, Huntly BJ. Molecular landscape of acute myeloid leukemia in younger adults and its clinical relevance. Blood. 2016;127(1):29-41.

13. Ramos NR, Mo CC, Karp JE, Hourigan CS. Current approaches in the treatment of relapsed and refractory acute myeloid leukemia. J Clin Med. 2015;4(4):665-695

14. Cocciardi S, Dolnik A, Kapp-Schwoerer S, et al. Clonal evolution patterns in acute myeloid leukemia with NPM1 mutation. Nat Commun. 2019;10(1):2031

15. McNeer NA, Philip J, Geiger H, et al. Genetic mechanisms of primary chemotherapy resistance in pediatric acute myeloid leukemia. Leukemia. 2019; 33(8): 1934-1943.
16. Onecha E, Linares M, Rapado I, et al. A novel deep targeted sequencing method for minimal residual disease monitoring in acute myeloid leukemia. Haematologica. 2019;104(2):288-296.

17. Cedena MT, Rapado I, Santos-Lozano A, et al. Mutations in the DNA methylation pathway and number of driver mutations predict response to azacitidine in myelodysplastic syndromes. Oncotarget. 2017;8(63):106948 106961

18. Gorello P, Cazzaniga G, Alberti F, et al. Quantitative assessment of minimal residual disease in acute myeloid leukemia carrying nucleophosmin (NPM1) gene mutations. Leukemia. 2006;20(6):1103-1108.

19. Burge C, Karlin S. Prediction of complete gene structures in human genomic DNA. J Mol Biol. 1997;268(1):78-94.

20. Rubio-Camarillo M, Lopez-Fernandez $H$, Gomez-Lopez G, et al. RUbioSeq+: a multiplatform application that executes parallelized pipelines to analyse next-generation sequencing data. Comput Methods Programs Biomed. 2017;138:73-81.

21. Cheson BD, Bennett JM, Kopecky KJ, et al. Revised recommendations of the International Working Group for diagnosis, standardization of response criteria, treatment outcomes, and reporting standards for therapeutic trials in acute myeloid leukemia. J Clin Oncol. 2003;21(24):4642-4649.

22. Dohner H, Estey EH, Amadori S, et al. Diagnosis and management of acute myeloid leukemia in adults: recommendations from an international expert panel, on behalf of the European LeukemiaNet. Blood. 2010;115(3):453-474

23. Christen F, Hoyer K, Yoshida $\mathrm{K}$, et al Genomic landscape and clonal evolution of acute myeloid leukemia with $t(8 ; 21)$ : an international study on 331 patients. Blood. 2019:133(10):1140-1151.

24. Jones JR, Weinhold N, Ashby C, et al. Clonal evolution in myeloma: the impact of maintenance lenalidomide and depth of response on the genetics and sub-clonal structure of relapsed disease in uniformly treated newly diagnosed patients. Haematologica. 2019; 104(7):1440-1450

25. Walter RB, Othus M, Burnett AK, et al. Resistance prediction in AML: analysis of 4601 patients from MRC/NCRI, HOVON/SAKK, SWOG and MD Anderson Cancer Center. Leukemia. 2015; 29(2):312320.

26. Rothenberg-Thurley M, Amler S, Goerlich $\mathrm{D}$, et al. Persistence of pre-leukemic clones during first remission and risk of relapse in acute myeloid leukemia. Leukemia. 2018;32(7):1598-1608.
27. Shlush LI. Age-related clonal hematopoiesis. Blood. 2018;131(5):496-504.

28. Bejar R. What biologic factors predict for transformation to AML? Best Pract Res Clin Haematol. 2018;31(4):341-345

29. Metzeler KH, Herold T, Rothenberg-Thurley $\mathrm{M}$, et al. Spectrum and prognostic relevance of driver gene mutations in acute myeloid leukemia. Blood. 2016;128(5):686-698.

30. Corces-Zimmerman MR, Hong WJ, Weissman IL, Medeiros BC, Majeti R. Preleukemic mutations in human acute myeloid leukemia affect epigenetic regulators and persist in remission. Proc Natl Acad Sci U S A. 2014;111(7):2548-2553.

31. Sottoriva A, Kang H, Ma Z, et al. A Big Bang model of human colorectal tumor growth. Nat Genet. 2015;47(3):209-216.

32. Jan M, Majeti R. Clonal evolution of acute leukemia genomes. Oncogene. 2013 32(2):135-140

33. Kronke J, Bullinger L, Teleanu V, et al. Clona evolution in relapsed NPM1-mutated acute myeloid leukemia. Blood. 2013; 122(1):100108.

34. Jaiswal S, Fontanillas P, Flannick J, et al. Agerelated clonal hematopoiesis associated with adverse outcomes. N Engl J Med. 2014;371(26):2488-2498.

35. Klco JM, Spencer DH, Miller CA, et al Functional heterogeneity of genetically defined subclones in acute myeloid leukemia. Cancer Cell. 2014;25(3):379-392.

36. Quek L, Ferguson P, Metzner $M$, et al. Mutational analysis of disease relapse in patients allografted for acute myeloid leukemia. Blood Adv. 2016;1(3):193-204

37. Shimizu $\mathrm{H}$, Yokohama A, Ishizaki T, et al Clonal evolution detected with conventional cytogenetic analysis is a potent prognostic factor in adult patients with relapsed AML. Hematol Oncol. 2018;36(1):252-257.

38. Marcucci G, Mrozek K, Ruppert AS, et al. Abnormal cytogenetics at date of morphologic complete remission predicts short overall and disease-free survival, and higher relapse rate in adult acute myeloid leukemia: results from cancer and leukemia group $\mathrm{B}$ study 8461. J Clin Oncol. 2004;22(12):24102418

39. Chen Y, Cortes J, Estrov Z, et al. Persistence of cytogenetic abnormalities at complete remission after induction in patients with acute myeloid leukemia: prognostic significance and the potential role of allogeneic stem-cell transplantation. J Clin Oncol. 2011;29(18):2507-2513.

40. Derman BA, Larson RA. Post-remission therapy in acute myeloid leukemia: Are we ready for an individualized approach? Best Pract Res Clin Haematol. 2019;32(4): 101102. 